\title{
The Sloan Digital Sky Survey Quasar Catalog V. Seventh Data Release
}

\author{
Donald P. Schneider, ${ }^{1}$ Gordon T. Richards, ${ }^{2}$ Patrick B. Hall,${ }^{3}$ Michael A. Strauss, ${ }^{4}$ \\ Scott F. Anderson, ${ }^{5}$ Todd A. Boroson, ${ }^{6}$ Nicholas P. Ross, ${ }^{1}$ Yue Shen,${ }^{4}$ W.N. Brandt,${ }^{1}$ \\ Xiaohui Fan, ${ }^{7}$ Naohisa Inada, ${ }^{8}$ Sebastian Jester, ${ }^{9,10}$ G.R. Knapp ${ }^{4}$ Coleman M. Krawczyk, ${ }^{2}$ \\ Anirudda R. Thakar, ${ }^{11}$ Daniel E. Vanden Berk, ${ }^{1}$ Wolfgang Voges,${ }^{12,13}$ Brian Yanny, ${ }^{14}$ \\ Donald G. York, ${ }^{15,16}$ Neta A. Bahcall, ${ }^{4}$ Dmitry Bizyaev,${ }^{17}$ Michael R. Blanton, ${ }^{18}$ \\ Howard Brewington, ${ }^{17}$ J. Brinkmann, ${ }^{17}$ Daniel Eisenstein, ${ }^{7}$ Joshua A. Frieman, ${ }^{19,14,15}$ \\ Masataka Fukugita, ${ }^{20}$ Jim Gray, ${ }^{21}$ James E. Gunn, ${ }^{4}$ Pascale Hibon, ${ }^{22}$ Željko Ivezić,${ }^{5}$ \\ Stephen M. Kent, ${ }^{14,15}$ Richard G. Kron, ${ }^{15,14}$ Myung Gyoon Lee, ${ }^{23}$ Robert H. Lupton, ${ }^{4}$ \\ Elena Malanushenko, ${ }^{17}$ Viktor Malanushenko, ${ }^{17}$ Dan Oravetz,${ }^{17}$ K. Pan, ${ }^{17}$ Jeffrey R. Pier, ${ }^{24}$ \\ Ted N. Price III, ${ }^{4}$ David H. Saxe ${ }^{25}$ David J. Schlegel, ${ }^{26}$ Audry Simmons,${ }^{17}$ \\ Stephanie A. Snedden, ${ }^{17}$ Mark U. SubbaRao, ${ }^{27}$ Alexander S. Szalay,${ }^{11}$ and \\ David H. Weinberg ${ }^{28}$
}




\footnotetext{
${ }^{1}$ Department of Astronomy and Astrophysics, The Pennsylvania State University, 525 Davey Laboratory, University Park, PA 16802.

${ }^{2}$ Department of Physics, Drexel University, 3141 Chestnut Street, Philadelphia, PA 19104.

${ }^{3}$ Department of Physics \& Astronomy, York University, 4700 Keele Street, Toronto, Ontario, M3J 1P3, Canada.

${ }^{4}$ Princeton University Observatory, Peyton Hall, Princeton, NJ 08544.

${ }^{5}$ Department of Astronomy, University of Washington, Box 351580, Seattle, WA 98195.

${ }^{6}$ National Optical Astronomy Observatory, Tucson, AZ 85726.

${ }^{7}$ Steward Observatory, University of Arizona, 933 North Cherry Avenue, Tucson, AZ 85721.

${ }^{8}$ Cosmic Radiation Laboratory, RIKEN, 2-1 Hirosawa, Wako, Saitama 351-0198, Japan.

${ }^{9}$ School of Physics and Astronomy, University of Southampton, Southampton SO17 1BJ, UK.

${ }^{10}$ Max-Planck-Institut für Astronomie, Königstuhl 17, D-69117 Heidelberg, Germany.

${ }^{11}$ Department of Physics and Astronomy, The Johns Hopkins University, 3400 North Charles Street, Baltimore, MD 21218-2686.

${ }^{12}$ Max-Planck-Institut für extraterrestische Physik, Postfach 1312, D-85741 Garching, Germany.

${ }^{13}$ Max Planck Digital Library, Amalienstrasse 33, D-80799, München, Germany.

${ }^{14}$ Fermi National Accelerator Laboratory, P.O. Box 500, Batavia, IL 60510.

${ }^{15}$ Department of Astronomy and Astrophysics, The University of Chicago, 5640 South Ellis Avenue, Chicago, IL 60637.

${ }^{16}$ Enrico Fermi Institute, The University of Chicago, 5640 South Ellis Avenue, Chicago, IL 60637.

${ }^{17}$ Apache Point Observatory, P.O. Box 59, Sunspot, NM 88349.

${ }^{18}$ Department of Physics, New York University, 4 Washington Place, New York, NY 10003.

${ }^{19}$ Center for Cosmological Physics, The University of Chicago, 5640 South Ellis Avenue Chicago, IL 60637.

${ }^{20}$ Institute for Cosmic Ray Research, University of Tokyo, 5-1-5 Kashiwa, Kashiwa City, Chiba 277-8582, Japan.

${ }^{21}$ Microsoft Research, 301 Howard Street, No. 830, San Francisco, CA 94105.

${ }^{22}$ Korean Institute for Advanced Study, 207-43 Cheongryangri-dong, Dongdaemun-gu, Seoul 130-012, Korea.

${ }^{23}$ Department of Physics and Astronomy, Seoul National University, Seoul 151-742, Korea.

${ }^{24}$ Division of Astronomical Sciences, National Science Foundation, 4201 Wilson Blvd, Arlington, VA 22230.

${ }^{25} 490$ Wilson's Crossing Road, Auburn, NH 03032.

${ }^{26}$ LBNL, Bldg 50R5032 MS 50-232, 1 Cyclotron Rd., Berkeley CA 94720-8160.
} 


\begin{abstract}
We present the fifth edition of the Sloan Digital Sky Survey (SDSS) Quasar Catalog, which is based upon the SDSS Seventh Data Release. The catalog, which contains 105,783 spectroscopically confirmed quasars, represents the conclusion of the SDSS-I and SDSS-II quasar survey. The catalog consists of the SDSS objects that have luminosities larger than $M_{i}=-22.0$ (in a cosmology with $H_{0}=70 \mathrm{~km} \mathrm{~s}^{-1} \mathrm{Mpc}^{-1}, \Omega_{M}=0.3$, and $\left.\Omega_{\Lambda}=0.7\right)$, have at least one emission line with FWHM larger than $1000 \mathrm{~km} \mathrm{~s}^{-1}$ or have interesting/complex absorption features, are fainter than $i \approx 15.0$, and have highly reliable redshifts. The catalog covers an area of $\approx 9380 \mathrm{deg}^{2}$. The quasar redshifts range from 0.065 to 5.46 , with a median value of 1.49; the catalog includes 1248 quasars at redshifts greater than four, of which 56 are at redshifts greater than five. The catalog contains 9210 quasars with $i<18$; slightly over half of the entries have $i<19$. For each object the catalog presents positions accurate to better than $0.1^{\prime \prime}$ rms per coordinate, five-band (ugriz) CCD-based photometry with typical accuracy of 0.03 mag, and information on the morphology and selection method. The catalog also contains radio, near-infrared, and X-ray emission properties of the quasars, when available, from other large-area surveys. The calibrated digital spectra cover the wavelength region $3800-9200 \AA$ at a spectral resolution of $\simeq 2000$; the spectra can be retrieved from the SDSS public database using the information provided in the catalog. Over $96 \%$ of the objects in the catalog were discovered by the SDSS. We also include a supplemental list of an additional 207 quasars with SDSS spectra whose archive photometric information is incomplete.
\end{abstract}

Subject headings: catalogs, surveys, quasars:general

\title{
1. Introduction
}

This paper describes the Fifth Edition of the Sloan Digital Sky Survey (SDSS; York et al. 2000) Quasar Catalog. Previous versions of the catalog (Schneider et al. 2002, 2003, 2005, 2007; hereafter Papers I, II, III, and IV) were published with the SDSS Early Data

\footnotetext{
${ }^{27}$ University of Chicago and Adler Planetarium and Astronomy Museum, 1300 S. Lake Shore Drive, Chicago, IL 60605.

${ }^{28}$ Department of Astronomy, Ohio State University, 140 West 18th Avenue, Columbus, OH 43210-1173.
} 
Release (EDR; Stoughton et al. 2002), the SDSS First Data Release (DR1; Abazajian et al. 2003), the SDSS Third Data Release (DR3; Abazajian et al. 2005), and the SDSS Fifth Data Release (DR5; Adelman-McCarthy et al. 2007), and contained 3,814, 16,713, 46,420, and 77,429 quasars, respectively. The current catalog is the entire set of quasars from the SDSS-I and SDSS-II Quasar Survey; the SDSS-II was completed on 15 July 2008 and the Seventh Data Release (DR7; Abazajian et al. 2009) was made public on 31 October 2008. The catalog contains 105,783 quasars.

The bulk of the quasars were identified as part of the SDSS Legacy Survey, which consisted of a systematic program to obtain spectra of one million galaxies and 100,000 quasars in two regions: an approximately 7,600 $\mathrm{deg}^{2}$ area centered on the North Galactic Pole (hereafter this region will be referred to as the North Galactic Cap) and approximately $800 \operatorname{deg}^{2}$ in three narrow regions in the proximity of the Celestial Equator centered near $0^{\circ}$ in right ascension (see York et al. 2000 and Abazajian et al. 2009). With this catalog, the spectroscopy in the North Galactic Cap region is now contiguous. Outside of the Legacy area quasars were found, frequently serendipitously, on a series of "Special Plates" (see Adelman-McCarthy et al. 2006) that were observed when Legacy observations could not be performed. A large fraction of the Special Plates were obtained as part of two programs: Extensions of the Legacy target selection algorithms along the Fall equatorial region (Adelman-McCarthy et al. 2006), and the Sloan Extension for Galactic Understanding and Exploration (SEGUE; Yanny et al. 2009), a project to study stellar populations in the Galactic halo. The distribution of the DR7 quasars on the celestial sphere is displayed in Figure 1.

The catalog in the present paper consists of quasars that have a luminosity larger than $M_{i}=-22.0$ (calculated assuming an $H_{0}=70 \mathrm{~km} \mathrm{~s}^{-1} \mathrm{Mpc}^{-1}, \Omega_{M}=0.3, \Omega_{\Lambda}=0.7$ cosmology, which will be used throughout this paper). The objects are denoted in the catalog by their DR7 J2000 coordinates; the format for the object name is SDSS Jhhmmss.ss+ddmmss.s. Since the image data used for the astrometric information can change between data releases (e.g., a region with poor seeing that is included in an early release is superseded by a newer observation in good seeing), the coordinates for an object can be modified at the $\approx 0.1^{\prime \prime}$ level (although on rare occasions (see $\S 6$ ) the change in position can exceed $1^{\prime \prime}$ ); hence the designation of a given source can change between data releases. When merging SDSS Quasar Catalogs with previous databases one should always use the coordinates, not object names, to identify unique entries.

Recent additional compilations of quasars based upon SDSS data include the DR5 Broad Absorption Line (BAL) Quasar Catalog (Gibson et al. 2009), the Two Degree FieldSDSS Luminous Red Galaxy and Quasar Survey (2SLAQ) spectroscopic catalog (Croom et al. 2009), and a sample of approximately one million "photometric" quasars (i.e., without 
spectroscopic confirmation) selected based upon their colors (Richards et al. 2009) drawn from the SDSS Data Release 6 (DR6; Adelman-McCarthy et al. 2008).

The DR7 catalog does not include classes of Active Galactic Nuclei (AGN) such as Type 2 quasars, Seyfert galaxies (i.e., low-luminosity AGN), and BL Lacertae objects. Recent studies of these sources in the SDSS can be found in Reyes et al. (2008) (Type 2), Hao et al. (2005) (Seyferts), and Plotkin et al. (2010) (BL Lacs). No emission line equivalent width limit is imposed for inclusion in the present catalog; there are some quasars with low equivalent width emission lines in the catalog, and they tend to be among the brighter objects (the higher the quality of the spectra, the easier the identification of small equivalent width features), or at relatively high redshift, where redshifts can be measured using absorption from the Lyman $\alpha$ forest (e.g., Fan et al. 1999). The highest redshift SDSS quasars $(z>5.7$; e.g., Fan et al. 2003, 2006; Jiang et al. 2009) were identified as candidates in the SDSS imaging data, but the spectra were not obtained with the SDSS spectrographs, so they are not included in the catalog.

The basic format and analysis of this work closely follow that of the previous four editions of the SDSS Quasar Catalog. The observations used to produce the catalog are presented in Section 2; the construction of the catalog and the catalog format are discussed in Sections 3 and 4, respectively. Section 5 presents an overview of the catalog, Section 6 contains a discussion of some issues relevant to statistical samples, and a summary is given in Section 7. The catalog is presented in an electronic table in this paper and can also be found at an SDSS public web site.29

\section{Observations}

The Sloan Digital Sky Survey used a CCD camera (Gunn et al. 1998) on a dedicated 2.5-m telescope (Gunn et al. 2006) at Apache Point Observatory, New Mexico, to obtain images in five broad optical bands (ugriz; Fukugita et al. 1996) over approximately 10,000 deg ${ }^{2}$ of the high Galactic latitude sky. The survey data-processing software measures the properties of each detected object in the imaging data in all five bands, and determines and applies both astrometric and photometric calibrations (Pier et al., 2003; Hogg et al. 2001; Lupton et al. 2001; Ivezić et al. 2004). The details of the SDSS photometric calibration are described in Smith et al. (2002), Tucker et al. (2006), and Padmanabhan et al. (2008). The SDSS photometric system is based on the AB magnitude scale (Oke \& Gunn 1983); the photometric measurements are reported as asinh magnitudes (Lupton, Gunn, \& Sza-

${ }^{29}$ http://www.sdss.org/dr7/products/value_added/qsocat_dr7.html 
lay 1999). Unlike previous editions of the SDSS quasar catalog, in this release we use the "ubercalibration" photometric calibrations of Padmanabhan et al. (2008).

The SDSS quasar target selection is described in Richards et al. (2002, hereafter GTR02) and in papers I-IV; we provide a brief summary of the identification of candidates and the spectroscopic observations here. Most quasar candidates are selected based on their location in multidimensional SDSS color-space. The Point Spread Function (PSF) magnitudes are used for the quasar target selection, and the selection is based upon measurements that have been corrected for Galactic extinction using the maps of Schlegel, Finkbeiner, \& Davis (1998). An $i$ magnitude limit of 19.1 is imposed for candidates whose colors indicate a probable redshift of less than $\approx 3$ (selected from the ugri color cube); high-redshift candidates (mainly selected from the griz color cube, but some $z<3.5$ are identified in the ugri color cube; see GTR02) are accepted if $i<20.2$ and the source is unresolved. In addition to the multicolor selection, unresolved objects brighter than $i=19.1$ that lie within 2.0" of a FIRST radio source (Becker, White, \& Helfand 1995) are also identified as primary quasar candidates. Target selection also imposes a maximum brightness limit $(i \approx 15.0)$ on quasar candidates to avoid saturation and cross-talk in the spectra.

The primary sample described above was supplemented by quasars that were targeted by the following SDSS spectroscopic target selection algorithms: Galaxy and Luminous Red Galaxy (Strauss et al. 2002 and Eisenstein et al. 2001), X-ray (object near the position of a ROSAT All-Sky Survey [RASS; Voges et al. 1999, 2000] source; see Anderson et al. 2003), Star (point source with a color typical of an interesting class of star), or Serendipity (unusual color or FIRST matches). The SDSS is designed to be complete in the Galaxy, Luminous Red Galaxy, and Quasar programs, but no attempt at completeness was made for the other three categories. Figure 2 shows the locations of stars and quasars in the SDSS color-color space (the quasar measurements are from objects in the DR7 catalog).

The final quasar selection algorithm of GTR02 was refined using the results of early SDSS observations, so not all objects in this catalog were selected using the GTR02 approach; all quasars originally reported in Papers I and II, and some in Paper III, were identified with pre-GTR02 methods. The catalog reports two target selection flags for each object: 1) TARGET, which is the flag that was used, based upon the imaging information and the selection algorithm available at the time, to select the quasar for the spectroscopic sample, and 2) BEST, which is the flag produced with the GTR02 algorithm and the latest available photometric information. Care must be exercised when constructing statistical samples from this catalog; see GTR02, Vanden Berk et al. (2005), and Richards et al. (2006) for discussions of survey completeness and efficiency, and the issues that are important for the construction of statistical SDSS quasar samples. 
In addition to the quasars found in the Legacy Survey, the catalog contains many quasars whose spectra were obtained on Special Plates (see Adelman-McCarthy et al. 2006). The main source of Special Plate quasars is the set of observations along the celestial equator in the Southern Galactic Cap ("Stripe 82"; see Stoughton et al. 2002) taken for a variety of commissioning and science purposes; for example, exploring the variations in the selection criteria of spectroscopic targets and empirically determining the completeness of the quasar survey (e.g., Vanden Berk et al. 2005). Another major component of the Special Plates are the observations taken for the SEGUE project (Yanny et al. 2009); these plates usually lie along lines of constant Galactic longitude and reach closer to the Galactic plane than do the Legacy Survey fields (see Figure 1). The SEGUE program required SDSS imaging outside of the Legacy Survey area.

The spectroscopic targets identified by the various SDSS selection algorithms are arranged onto a series of $3^{\circ}$ diameter circular fields (Blanton et al. 2003). The two SDSS double spectrographs produce data covering 3800-9200 $\AA$ at a spectral resolution of $\simeq 2000$; a dichroic splits the beam at $6150 \AA$. The data, along with the associated calibration frames, are processed by the SDSS spectroscopic pipeline (see Stoughton et al. 2002). Several improvements have been made to the spectroscopic software since DR5, in particular the wavelength calibration, spectrophotometric calibration, and the handling of strong, unresolved emission features (see Adelman-McCarthy et al. 2008 and Abazajian et al 2009 for a description of these modifications).

The calibrated spectra are classified into various groups (e.g., star, galaxy, quasar), and redshifts are determined by two independent software packages: spectro1d (see $\S 4.10 .2 .1$ of Stoughton et al. 2002), which assigns absorption-line redshifts by cross-correlating the observations with a series of template spectra in Fourier space and Gaussian fits to emission lines to measure emission-line redshifts; and specBS (Adelman-McCarthy et al. 2008), which performs $\chi^{2}$ fits in wavelength space between the observations and templates. The quality of the redshift is quantified by spectro1d by a parameter called the "Confidence Limit", which is stored in the parameter zconf (Stoughton et al. 2002). A large value of zconf usually indicates a solid redshift measurement, but a low value does not necessarily mean an uncertain redshift; see $\S 6$ for a discussion of this important issue. The catalog reports the spectro1d redshifts, which have typical quoted redshift errors of $\approx 0.004$ (except in the cases where visual inspection found that the spectro1d redshift was incorrect; see §3.2). Hewett \& Wild (2010) present evidence for systematic errors of $\approx 0.003$ in SDSS quasar redshifts measurements; these errors are small for most applications, but can be significant for some investigations.

The catalog contains photometry from 346 SDSS imaging runs acquired between 19 Septem- 
ber 1998 (Run 94) and 1 January 2008 (Run 7264), and spectra from 2210 spectroscopic plates taken between 5 March 2000 and 6 July 2008. Figure 3 shows the calibrated SDSS spectra of four catalog quasars, added since Paper IV, representing a range of properties. The processed DR7 spectra have not been corrected for Galactic extinction.

\section{Construction of the SDSS DR7 Quasar Catalog}

As described above, the quasars in the catalog were drawn from two sets of SDSS observations: the Legacy Survey area, and the Special Plates, where the spectroscopic targets were not chosen by the standard SDSS target selection algorithms. The DR7 quasar catalog was constructed in three stages: 1) creation of a quasar candidate database, 2) visual examination of the spectra of the quasar candidates, and 3) application of luminosity and emission-line velocity width criteria. This approach is similar to that used in Papers I-IV, but with minor modifications.

This catalog of bona-fide quasars, that have redshifts checked by eye and luminosities and line widths that meet the formal quasar definition, is constructed from a larger "master" table of quasar candidates and confirmed quasars. This master table was created using an SQL query to the public SDSS-DR7 database (i.e., the Catalog Archive Server [CAS]; see Thakar et al 2008 and http://cas.sdss.org/astrodr7/). The vast majority of the objects in the master table were found from the union of four classes of objects: 1) those targeted, by either TARGET or BEST, by the quasar selection algorithm (GTR02) based upon their photometric or radio properties, 2) those whose spectra were classified by spectro1d as quasars (specClass=QSO or HIZ_QSO), 3) those whose spectra are classified as UNKNOWN by spectro1d, and 4) any object whose spectro1d redshift is larger than 0.6. (There is of course considerable overlap between these categories.) The spectra in the third category often have low $\mathrm{S} / \mathrm{N}$ or some glaring instrument-induced defects, but the set of UNKNOWN objects also include a considerable number of quasars that would have been missed by the other three selection criteria (e.g., extreme BALs; Hall et al. 2002). The final category recovered the few quasars that were not selected by the GTR02 algorithm but whose spectra were misclassified by spectro1d, usually as galaxies. The lower redshift limit of criterion (4) was imposed because of the rapid rise in the number of objects as the redshift limit was decreased. A search of the specBS database found 112 quasars that had not been identified by the SQL query; these objects were added to the master quasar list.

Comparison of a preliminary master table to the 77,429 quasars included in Paper IV revealed that a small number of quasars were missing, mostly due to changes in adopted redshifts or classifications. The following subsets were added to the master table, using 
purely heuristic criteria designed to maximize the recovery of known missing objects while minimizing the inclusion of large numbers of objects that were not quasars.

- Objects whose cross-correlation with quasar templates in the spectro1d processing yielded $z>0.6$, but for which the CAS entry is a higher-confidence spectro1d redshift below 0.6 based upon fitting to emission lines, and with a confidence on that cross-correlation redshift which is at least $80 \%$ of the CAS zConf value.

- Objects whose spectro1d emission-line redshift exceeded 0.6, but for which the CAS entry is a higher-confidence spectro1d redshift below 0.6 based upon cross-correlation with the quasar templates, and with a confidence on that emission-line cross-correlation redshift which is at least $80 \%$ of the CAS zConf value.

- Objects at $0.35<z<0.6$ which have a Mg II emission line detected at $\geq 3.5 \sigma$ significance with an observed linewidth (FWHM) of $\geq 5.4 \AA$ and a linewidth uncertainty of no more than $40 \%$.

- One Paper IV quasar is not in the DR7 CAS (the "unmapped fiber" object discussed in Paper IV); information on this object, taken from Paper IV, was manually added to the master table.

The final table contains information for 1,293,260 source 30 , of which 300,357 entries have spectroscopic observations. Multiple entries for a given object are retained in the master table. The following culls were then applied to this database: 1) Objects that were lacking spectra (992,903 entries), 2) Objects whose photometric measurements have not been loaded into the CAS, and thus luminosity calculations are impossible to perform (6383; 207 of these objects are indeed quasars and are described in Appendix A), 3) Candidates that were classified by spectro1d as SPEC_STAR or SPEC_SKY, with redshifts less than 0.002, and had the redshift status flag set to either XCORR_EMLINE, XCORR_HIC, EMLINE_XCORR, or EMLINE_HIC $(48,712)$, and 4) Multiple spectra (coordinate agreement better than 1.0") of the same object $(65,632)$.

Before proceeding, we should make a few brief comments on the last two items. Ten of the Paper IV quasars were classified by the DR7 spectroscopic pipeline as stars with high confidence. In these individual cases, we overrode the automatic rejection and retained the quasars in the catalog. There are undoubtedly a few additional quasars residing in this large subset of confidently classified stars, but the effort required to visually examine the nearly 50,000 spectra was not deemed to be worth the miniscule return of quasars. In cases of

\footnotetext{
${ }^{30}$ The master table is known as the QSOConcordanceAll table, which can be found in the SDSS database; see http://cas.sdss.org/astrodr7/en/help/browser/description. asp?n=QsoConcordanceAll $k t=U$.
} 
duplicate spectra of an object, the "science primary" spectrum is selected (i.e., the spectrum was obtained as part of normal science operations); when there is more than one science primary observation (or when none of the spectra have this flag set), the spectrum with the highest S/N is retained (see Stoughton et al. 2002 for a description of the science primary flag). These actions produced a list of 179,630 unique quasar candidates.

Given the considerable size of the DR7 quasar candidate list, our previous practice of visually examining every spectrum, irrespective of whether it had been reviewed for a previous edition of the catalog, was abandoned. Only those objects whose DR7 spectra (identified by the Modified Julian Date of observation, plate number, and fiber number) were not in the Paper IV list were by default reviewed (55,666 spectra). We also visually examined the spectra for the 1,224 quasars whose Paper IV redshifts differed by the DR7 spectro1d values by more than 0.03 .

The SDSS spectra of these quasar candidates were manually inspected by several of the authors; as in previous papers in this series, we found that the spectroscopic pipeline redshifts and classifications of the overwhelming majority of the objects are accurate. The redshifts for 2671 of the quasar candidates were manually adjusted, usually by significant (several tenths or more) amounts; the main cause for revision, as one would expect, was that spectro1d assigned the incorrect identification to emission features.

The absolute magnitude limit for inclusion in the catalog is $M_{i}=-22.0$, calculated by correcting the BEST $i$ PSF magnitude measurement for Galactic extinction (using the maps of Schlegel, Finkbeiner, \& Davis 1998) and assuming that the quasar spectral energy distribution in the ultraviolet-optical can be represented by a power law $\left(f_{\nu} \propto \nu^{\alpha}\right)$, where $\alpha=-0.5$ (Vanden Berk et al. 2001). In the 20 cases where BEST $i$ photometry was unavailable, the TARGET measurements were substituted for the absolute magnitude calculation. (There are 52 quasars that lack TARGET photometry.)

This approach ignores the contributions of emission lines and the observed distribution in continuum slopes to the photometric measurements. Emission lines can contribute several tenths of a magnitude to the k-correction (see Richards et al. 2006), but since there is essentially no emission line contribution to the $i$ flux at zero redshift, our adopted approach will be inclusive (i.e., if one wishes to apply a k-correction for emission lines to construct a sample of objects with continuum luminosities greater than or equal to $M_{i}=-22.0$, our luminosity cut will not have removed any objects that satisfy the emission-line k-corrected sample).

Quasars near the $M_{i}=-22.0$ luminosity limit are often of similar brightness in the $i$-band as the starlight produced by the host galaxy. Although the PSF-based SDSS pho- 
tometric data presented in the catalog are less susceptible to host galaxy contamination than are fixed-aperture measurements, the nucleus of the host galaxy can still contribute appreciably to this measurement for the lowest luminosity entries in the catalog (e.g., Hao et al. 2005, Bentz et al. 2006, Croom et al. 2009).

The visual inspection and application of the luminosity criterion reduced the number of quasar candidates to under 106,000 objects. During the visual inspection we included all spectra that might possess at least one broad line, but no quantitative linewidth measurements were made. To remove narrow-line objects, the spectra of the candidate quasars were examined with the Principal Component Analysis (PCA) code of Boroson \& Lauer (2010). The PCA analysis was done by constructing eigenspectra from approximately 1000 of the highest S/N spectra after rejecting the few narrow-lined objects in this subset.

This procedure was applied separately to the $4300-5400 \AA$ rest frame region of the 15,900 objects with $z<0.7$ and to the $2400-3200 \AA$ rest frame region of the 55,400 objects with $0.7<z<1.8$. The spectra that could not be fit with a small number (15) of these eigenspectra were visually examined. Almost all of the 120 rejects were narrow-line objects, with about $75 \%$ being Seyfert 2s or LINERS, and the remainder were spectra with H IIregion line ratios. We also examined the spectra of sources that were located near the highest concentration of narrow-line objects in the eigenspace; this exercise identified a handful of additional objects to reject. This process also uncovered a few objects with incorrect redshifts, which we revised.

Several of the high-redshift $(z>0.7)$ quasar candidates have images that appear extended, as quantified by the difference between their PSF and model magnitudes (see, e.g., the discussion in Stoughton et al. 2002; Strauss et al. 2002). We examined in detail the spectra and images of all $z>0.7$ quasar candidates with $r$ band PSF - model magnitudes greater than $0.2 \mathrm{mag}$. Many of these objects are quasar-galaxy superpositions, and the sample includes previously known gravitational lenses (e.g., Inada et al. 2010; McGreer et al. 2010). There were also 24 objects which on closer inspection were not quasars, and they were removed from the sample.

\section{Catalog Format}

The DR7 SDSS Quasar Catalog is available in three types of files at the SDSS public web site listed in the introduction: 1) a standard ASCII file with fixed-size columns, 2) a gzipped compressed version of the ASCII file, and 3) a binary FITS table format. The following description applies to the standard ASCII file. All files contain the same number 
of columns, but the storage of the numbers differs slightly in the ASCII and FITS formats; the FITS header contains all of the required documentation.

The standard ASCII catalog (Table 2 of this paper), which is $52 \mathrm{MB}$ in size, has a format that differs in only minor ways from that used in Paper IV. The first 80 lines consist of catalog documentation; this material is followed by 105,783 lines, each containing 74 columns. A summary of the information is given in Table 1 (the documentation in the ASCII catalog header is essentially an expansion of Table 1). At least one space separates all the column entries, and, except for the first and last columns (SDSS designation and the object name if previously known), all entries are reported in either floating point or integer format.

A summary of the spectroscopic selection, for both the TARGET and the BEST algorithms, is given in Table 3. We report seven selection classes in the catalog (columns 38 to 44 for BEST, 55-61 for TARGET). Each selection version has two columns, the number of objects that satisfied a given selection criterion and the number of objects that were identified only by that selection class. Of the 70,365 DR7 quasars that have Galactic-absorption corrected TARGET $i$ magnitudes brighter than 19.1, 68,497 (97.3\%) were identified by the TARGET quasar multicolor selection; if one combines TARGET multicolor and FIRST selection (the primary quasar target selection criteria), all but 1150 of the $i<19.1$ objects were selected. (The spectra of many of the last category of objects were obtained in observations that were not part of the primary quasar survey, i.e., the Special Plates.) The numbers are similar if one uses the BEST photometry and selection, although the completeness is not quite as high as with TARGET values.

\section{Catalog Summary}

The 105,783 objects in the catalog represent an increase of 28,354 quasars over the Paper IV database; of the entries in the new catalog, 101,945 (96.4\%) were either not listed in the NASA/IPAC Extragalactic Database (NED) or were recorded as an SDSS discovery in the NED database. (NED occasionally lists the SDSS name for objects that were not discovered by the SDSS.) The catalog quasars span a wide range of properties: redshifts from 0.065 to $5.461,14.86<i<22.36$ (1341 objects have $i>20.5$; only 183 have $i>21.0$ ), and $-30.28<M_{i}<-22.00$. The catalog contains 8630, 5377, and 53,564 matches to the FIRST, RASS, and 2MASS data, respectively. The RASS and 2MASS catalogs cover essentially all of the DR7 area, but $6865(6.5 \%)$ of the entries in the catalog lie outside of the FIRST region.

Figure 4 displays the distribution of the DR7 quasars in the $i$-redshift plane. Objects 
denoted by NED as previously discovered by investigations other than the SDSS are indicated with open circles. The curved cutoff on the left hand side of the graph is produced by the minimum luminosity criterion $\left(M_{i}<-22.0\right)$. The ridge in the contours at $i \approx 19.1$ for redshifts below three reflects the flux limit of the low-redshift sample; essentially all of the large number of $z<3$ points with $i>19.1$ are quasars selected via criteria other than the primary multicolor sample.

The clear majority of quasars have redshifts below two (the median redshift is 1.49 , the mode is $\approx 1.85$ ), but there is a significant tail of objects extending to redshifts well beyond $z>4$ (see upper left panel in Figure 5). The catalog contains 152 quasars with redshifts below 0.15 (21 with $z<0.10$ ). All of the 152 objects are of low luminosity $\left(M_{i}>-24.0\right.$, only six have $M_{i}<-$ because of the $i \approx 15.0$ limit for the spectroscopic sample. About $70 \%$ of these quasars (106) are classified by the SDSS processing software as resolved in the SDSS image data. The images of 4392 of the quasars are classified as extended by the SDSS photometric pipeline; $4124(94 \%)$ have redshifts below one (there are ten resolved $z>3.0$ quasars). The spectrum of the lowest redshift object is displayed in Figure 3. The DR7 catalog contains 1248 quasars with redshifts larger than four; 56 entries have redshifts above five, and one of the post-DR5 quasars has a redshift of 5.461 (see Figure 3), which exceeds the previous record of 5.41 found in the SDSS spectroscopic survey (Anderson et al. 2001).

The $i$-magnitude distribution of the DR7 quasars is shown in the upper right panel of Figure 5. Although the spectroscopic survey is limited to objects fainter than $i \approx 15$, the SDSS discovered a number of "PG-class" (Schmidt \& Green 1983) objects (see Jester et al. 2005). The DR7 catalog contains 124 entries with $i<16.0 ; 19$ of the quasars lack a NED entry. As can be seen from the $M_{i}$ diagram (lower right panel in Figure 5), the SDSS quasar survey is composed primarily of fairly luminous quasars; the distribution is roughly symmetric around $M_{i}=-26$ with a FWHM of approximately one magnitude (in the adopted cosmology $3 \mathrm{C} 273$ has $M_{i} \approx-26.6$ ). The histogram declines sharply at high luminosities (only $1.6 \%$ of the objects have $\left.M_{i}<-28.0\right)$ and has a gradual decline toward lower luminosities. Of the 134 catalog quasars with $M_{i}<-29.0,84$ either have no NED entry or are identified as an SDSS quasar; the redshifts of these objects lie between 1.30 and 4.97. Six quasars have $M_{i}<-30.0$. The two most luminous quasars have virtually identical absolute magnitudes (-30.26 and -30.28): 2MASSI J0745217+473436 (= SDSS J074521.78+473436.2), at $z=3.22$, and HS 1700+6416 (= SDSS J170100.60+641209.3), at $z=2.735$.

It has long been known that the majority of optically-selected quasars inhabit a restricted range in photometric color, and the large sample size and accurate photometry of the SDSS revealed a tight color-redshift correlation for quasars (Richards et al. 2001, 2003, Paper IV). The modal relations for quasar colors in the four standard SDSS colors as a 
function of redshift are given in Paper IV; the differences between those values and the ones calculated from the DR7 sample are negligible. For each of the catalog quasars we provide the quantity $\Delta(g-i)$, which is defined by

$$
\Delta(g-i)=(g-i)_{\mathrm{QSO}}-\langle(g-i)\rangle_{\mathrm{redshift}}
$$

where $\langle(g-i)\rangle_{\text {redshift }}$ is the modal value of this color at the redshift of the quasar. This "differential color" provides an estimate of the relative continuum slope of the quasar (values above zero indicate that the object has a redder continuum than the typical quasar at that redshift). The distribution of $\Delta(g-i)$ near the modal curve is roughly symmetric, but the lower left panel in Figure 5 reveals a significant population of "red" quasars that has no "blue" counterpart (also see Richards et al. 2003 and York et al. 2006). The usefulness of this measurement is compromised at redshifts above three because of the impact of the Lyman $\alpha$ forest and Lyman-limit systems in the observed $g$ photometry. These color-redshift relations have led to considerable success in assigning photometric redshifts to quasars (e.g., Weinstein et al. 2004 and references therein).

A total of 8630 catalog objects are FIRST (Becker, White, and Helfand 1995) sources (defined by a SDSS-FIRST positional offset of less than 2.0"). Note that 336 of the objects were selected (with TARGET) solely because they were FIRST matches. Extended radio sources may be missed by this matching. The upper left panel in Figure 6 contains a histogram of the angular offsets between the SDSS and FIRST positions; the solid line is the expected distribution assuming a $0.20^{\prime \prime} 1 \sigma$ Gaussian error in the relative SDSS/FIRST positions (found by fitting only the points with a separation less than $1.0^{\prime \prime}$ ). The smallangle separations are well-fit to the Rayleigh distribution, but outside of about $0.5^{\prime \prime}$ there is an obvious excess of observed separations; this large-separation tail is presumably due to sources with extended and/or complex radio emission. Given the high astrometric accuracy of the SDSS and FIRST catalogs, the fraction of false matches is quite small (on the order of $0.1 \%$; see Paper IV).

Matches with the ROSAT All-Sky Survey Bright and Faint Source Catalogs (Voges et al. 1999,2000 ) were made with a maximum allowed positional offset of $30^{\prime \prime}$; this is similar to the 27.5" positional coincidence required for the SDSS ROSAT target selection code (Anderson et al. 2003). During the ROSAT all-sky survey phase, some regions in the sky received little or no exposure due to a malfunction of the satellite's attitude measuring and control system. These regions of the sky were reobserved - from 1997 February 4 to February 26 - by a set of 783 pointings (the satellite had lost its capability to perform survey mode operations) with typical exposures of 1500 s. Approximately 2900 X-ray source detections were obtained in these fields; 2708 are unique objects and are not contained in the bright and faint source 
catalogues of ROSAT. These supplemental sources were included in our ROSAT matching.

The catalog contains 5377 RASS matches; approximately $1.3 \%$ are expected to be false identifications (Paper IV). The SDSS-RASS offsets for these matches are presented in the upper right panel of Figure 6; the solid curve, which is the Rayleigh distribution for a $1 \sigma$ positional error of $10.89^{\prime \prime}$ (fit using all the points), matches the data quite well.

Infrared photometric information for the quasars was provided by the Two Micron All Sky Survey (2MASS; Skrutskie et al. 2006). The SDSS quasar positions were compared to the 2MASS All-Sky and "6x" point source catalogs using a matching radius of 2 ". (The 6x catalog covered a smaller area of sky six times deeper than the main survey.) If matches were found in both the All-Sky and 6x catalogs, the information in the latter was selected for the quasar catalog. In the rare (approximately 1.5\%) cases where this approach found more than one 2MASS match for an SDSS quasar in a given catalog, the 2MASS source with the smallest separation was adopted. This procedure identified 2MASS photometry for 13,930 of the quasars. This approach is similar to, but slightly more extensive, than the 2MASS matching in previous SDSS quasar catalogs.

Since the 2MASS catalogs represent only the most secure $(5 \sigma)$ sources, and our object positions are known to considerable accuracy, it is possible to obtain additional information by extracting photometric data from the 2MASS images themselves. We have performed standard aperture photometry $\left(2^{\prime \prime}\right.$ radius) on the 2MASS images, following the procedure outlined in Gallagher et al. (2008), and report all detections larger than $2 \sigma$ in the quasar catalog. (The " $2 \sigma$ " objects have the 2 MASS flag set to " 2 " in the quasar catalog.) A total of 39,634 quasars had $2 \sigma$ detections in at least one 2MASS band, bringing the total number of objects in the catalog with infrared photometry to 53,564. Of the 1171 quasars with $i<17$, almost all (1167) have a 2MASS $J$-band detection. The offsets between the SDSS and 2MASS positions are shown in the lower left panel of Figure 6. Only the matches with the 2MASS catalog are displayed in the graph.

The mechanical constraint that SDSS spectroscopic fibers must be separated by $55^{\prime \prime}$ on a given plate makes it difficult for the spectroscopic survey to confirm close pairs of quasars. In regions that are covered by more than one plate, however, it is possible to obtain spectra of both components of a close pair. There are 449 pairs of quasars in the catalog with angular separation less than an arcminute, of which 46 have angular separations less than $20^{\prime \prime}$. Most of the pairs are chance superpositions, but there are many sets whose components have similar redshifts, suggesting that the quasars may be physically associated (e.g., Hennawi et al. 2006). The typical uncertainty in the measured value of the redshift difference between two quasars is $\approx 0.015$; the catalog contains 27 quasar pairs with separations of less than an arcminute and with $\Delta z<0.02$. These pairs, which are excellent candidates for 
binary quasars, are listed in Table 4. One of the pairs, SDSS J140012.77+313454.1 and SDSS J140012.85+313452.7, at a redshift of 3.3 and a separation of $1.7^{\prime \prime}$, is known to be a gravitational lens (Inada et al. 2008).

\section{Discussion}

The DR5 database is entirely contained in that of DR7, but there are 181 quasars from Paper IV $(0.17 \%)$ that do not have a counterpart within 1.0" of a DR7 quasar. (This fraction is similar to the $0.15 \%$ dropped between Papers III and IV.) Of the 181, there are ten that are included in the DR7 list that match at larger offsets; eight have matches within $2^{\prime \prime}$ and the other two have separations of $2.0^{\prime \prime}$ and $5.3^{\prime \prime}$. These ten separations are much larger than one would expect to find based on the $0.1^{\prime \prime}$ rms scatter in the positions; the large coordinate offsets presumably arise from different versions of the imaging pipeline software (in particular the deblender).

The majority of the dropped objects are low-redshift, low-luminosity sources $(\approx 80 \%$ have redshifts below one). Most of the changes were introduced because 1) the spectra did not appear to satisfy our quasar criteria (primarily the minimum line width limit) based upon the PCA analysis or 2) small changes in the photometric measurements dropped the luminosity below our absolute magnitude criterion.

Although the quasars in the catalog have been selected using a variety of techniques (optical colors, radio properties, X-ray emission), the information contained in the cata$\log$ allows one to easily construct well-defined subsamples. For example, to construct an optically-selected set of quasars (e.g., Strateva et al. 2005), one can either require that the low-redshift or high-redshift target selection flag is set, or, alternatively, require that the object was not chosen solely by radio/X-ray criteria (depending on whether or not you wish to have AGN found by the serendipity, galaxy, or star algorithms), using the quantities in columns 55-61 in the catalog.

The primary purpose of this catalog is to compile all of the quasars discovered/recovered by the SDSS survey in a complete and robust manner, allowing the general user to avoid the pitfalls that can arise from data mining such a large repository. All of the spectra have been inspected by eye, frequently by more than one of the authors. It may be that other users desire a more inclusive list of AGNs; for example, by relaxing our cuts on luminosity or line width. In creating an expanded AGN list, it is important to consider two important issues in terms of the compilation and statistical analysis of such samples.

First, it is tempting to eliminate the labor-intensive visual examination stage and rely 
on the zconf flag as a means of restricting the AGN sample to the most robust objects. However, zconf is not a good measure of the reliability of quasar redshifts: it depends strongly on redshift, as different emission lines enter and leave the SDSS spectral coverage. For example, zconf drops dramatically in the mean from $z \sim 0.7$ to $z \sim 0.9$ as the $\mathrm{H} \beta$ feature leaves the SDSS spectral bandpass. The left panel of Figure 7 shows zconf as a function of redshift for bona-fide quasars whose spectra have been confirmed by eye. The red histogram in the right panel in Figure 7 demonstrates the result of applying an arbitrary zconf $>0.95$ cut, independent of redshift, to the DR7 quasar sample. The redshift dependence of zconf introduces an artificial apparent periodicity in the redshift distribution.

The second issue has to do with the effects of emission lines on quasar photometry. The SDSS quasar selection will include intrinsically fainter objects whenever a strong emission line enters the $i$ bandpass, making the quasar appear brighter than the same quasar at a redshift where the observed $i$ filter covers only continuum emission (Richards et al. 2006). When we restrict the sample to $i<19.1$ and correct for the emission line k-correction (green histogram in Figure 7), the redshift distribution of the DR7 quasars is quite smooth.

\section{Summary}

This is the final edition of the Sloan Digital Sky Survey I and II Quasar Catalog, which was compiled from observations taken between 1999 and 2008. One of the original stated goals of the SDSS was to obtain spectra of 100,000 quasars, a total that has been met with this catalog. The lower right panel in Figure 6 charts the progress of the SDSS Quasar Survey, denoted by the number of spectroscopically-confirmed quasars as a function of time, over the duration of the project.

In the summer of 2008 the third phase of the SDSS, denoted as SDSS-III, began; this survey is planned to be in operation for six years. One of the components of SDSS-III, the Baryonic Oscillation Spectroscopic Survey (BOSS; Schlegel, White, \& Eisenstein 2009), will use improved spectrographs on the SDSS telescope to obtain spectra of 1.5 million luminous galaxies and over $100,000 z \approx 2.5$ quasars. We plan to continue our policy of timely releases of high-quality quasar catalogs throughout the SDSS-III era.

We would like to thank Paul Hewett for his very helpful comments on an early version of the DR7 quasar catalog. This work was supported in part by National Science Foundation grants AST-0607634 (DPS, DVB, NPR), AST-0307384 (XF), and AST-0707266 (MAS and YS), and by NASA LTSA grant NAG5-13035 (WNB, DPS). GTR was supported in part by a Alfred P. Sloan Foundation Fellowship, and PBH acknowledges support by NSERC. XF 
acknowledges support from an Alfred P. Sloan Fellowship and a David and Lucile Packard Fellowship in Science and Engineering.

Funding for the SDSS and SDSS-II has been provided by the Alfred P. Sloan Foundation, the Participating Institutions, the National Science Foundation, the U.S. Department of Energy, the National Aeronautics and Space Administration, the Japanese Monbukagakusho, the Max Planck Society, and the Higher Education Funding Council for England. The SDSS Web site is http://www.sdss.org/.

The SDSS is managed by the Astrophysical Research Consortium (ARC) for the Participating Institutions. The participating institutions are the American Museum of Natural History, Astrophysical Institute of Potsdam, University of Basel, Cambridge University, Case Western Reserve University, University of Chicago, Drexel University, Fermilab, the Institute for Advanced Study, the Japan Participation Group, Johns Hopkins University, the Joint Institute for Nuclear Astrophysics, the Kavli Institute for Particle Astrophysics and Cosmology, the Korean Scientist Group, the Chinese Academy of Sciences (LAMOST), Los Alamos National Laboratory, the Max-Planck-Institute for Astronomy (MPIA), the MaxPlanck-Institute for Astrophysics (MPA), New Mexico State University, Ohio State University, University of Pittsburgh, University of Portsmouth, Princeton University, the United States Naval Observatory, and the University of Washington.

This research has made use of 1) the NASA/IPAC Extragalactic Database (NED) which is operated by the Jet Propulsion Laboratory, California Institute of Technology, under contract with the National Aeronautics and Space Administration, and 2) data products from the Two Micron All Sky Survey, which is a joint project of the University of Massachusetts and the Infrared Processing and Analysis Center/California Institute of Technology, funded by the National Aeronautics and Space Administration and the National Science Foundation.

\section{A. Quasars Lacking Catalog Archive Server Photometry}

We required that every quasar in our catalog had photometry available in the CAS (the photometry was required to determine if the object met our luminosity criterion). There were 6383 objects with spectra in the quasar master list without photometry, of which 207 are genuine quasars. A few of these quasars were missed due to archiving errors in matching the BEST, TARGET and spectroscopic data; the remainder are drawn from four "special" plates (see the DR4 paper, Adelman-McCarthy et al. 2006) whose photometry was not entered into the CAS in a consistent manner. Three of those plates (1468, 1471, and 1472) targetted objects with quasar colors from fields in the vicinity of M31 (Adelman-McCarthy 
et al. 2007); these quasars can be used to study absorption from gas in M31. A fourth plate, 797, selected F star candidates from a field in Stripe 82, but the matching to the photometry was never done properly.

The data for these objects are included in Table 5, which includes a subset of the data columns of the primary catalog. With two exceptions, these objects all have listed PSF photometry drawn from the Data Archive Server. The redshifts range from 0.148 to 3.18 , with a median of 1.5 , i.e., very similar to that of the primary catalog quasars. These quasars are not included in the summary figures in the paper. Table 5 contains the following information for each quasar: SDSS name, J2000 right ascension and declination (degrees), redshift, ugriz PSF magnitudes and errors (uncorrected for Galactic extinction), Galactic extinction in the $u$ filter (magnitudes), morphological type ( $0=$ star, $1=$ galaxy), Primary Target flag, photometric run number, rerun number, camera column, field and object ID, and the MJD, plate, and fiber values for the spectrum. 


\section{REFERENCES}

[Abazajian et al. 2003]Abazajian, K., et al. 2003, AJ, 126, 2081 (DR1)

[Abazajian et al. 2005]Abazajian, K., et al. 2005, AJ, 129, 1755 (DR3)

[Abazajian et al. 2009]Abazajian, K., et al. 2009, ApJS, 182, 543 (DR7)

[Adelman-McCarthy et al. 2006]Adelman-McCarthy, J., et al. 2006, ApJS, 162, 38 (DR4)

[Adelman-McCarthy et al. 2007]Adelman-McCarthy, J., et al. 2007, ApJS, 172, 634 (DR5)

[Adelman-McCarthy et al. 2008]Adelman-McCarthy, J., et al. 2008, ApJS, 175, 297 (DR6)

[Anderson et al. 2001] Anderson, S.F., et al. 2001, AJ, 122, 503

[Anderson et al. 2003] Anderson, S.F., et al. 2003, AJ, 126, 2209

[Barkhouse \& Hall 2001]Barkhouse, W.A., \& Hall, P.B. 2001, AJ, 121, 2843

[Becker et al 1995]Becker, R.H., White, R.L., \& Helfand, D.J. 1995, ApJ, 450, 559

[Blanton et al. 2003]Blanton, M.R., Lupton, R.H., Maley, F.M., Young, N., Zehavi, I., \& Loveday, J. 2003, AJ, 125, 2276

[Bentz et al 2006]Bentz, M.C., Peterson, B.M., Pogge, R.W., Vestergaard, M., \& Onken, C.A. 2006, ApJ, 644, 133

[Boroson \& Lauer 2010]Boroson, T.A., \& Lauer, T.R. 2010, AJ, in press

[Croom et al. 2009]Croom, S.C., et al. 2009, MNRAS, 392, 19

[Eisenstein et al. 2002]Eisenstein, D.J., et al. 2001, AJ, 122, 2267

[Fan et al. 1999]Fan, X., et al. 1999, ApJ, 526, L57

[Fan et al. 2003]Fan, X., et al. 2003, AJ, 125, 1649

[Fan et al. 2006]Fan, X., et al. 2006, AJ, 132, 171

[Fukugita et al. 1996]Fukugita, M., Ichikawa, T., Gunn, J.E., Doi, M., Shimasaku, K., \& Schneider, D.P. 1996, AJ, 111, 1748

[Gallagher et al 2007]Gallagher, S.C., Hines, D.C., Blaylock, M., Priddey, R.S., Brandt, W.N., and Egami, E.E. 2007, ApJ, 665, 157 
[Gibson et al. 2009]Gibson, R.R., et al. 2009, ApJ, 692, 758

[Gunn et al. 1998]Gunn, J.E., et al. 1998, AJ, 116, 3040

[Gunn et al. 2006]Gunn, J.E., et al. 2006, AJ, 131, 2332

[Hall et al. 2002]Hall, P.B., et al. 2002, ApJS, 141, 267

[Hao et al. 2005]Hao, L., et al. 2005, AJ, 129, 1783

[Hennawi et al. 2006]Hennawi, J., et al. 2006, AJ, 131, 1

[Hennawi et al. 2010]Hennawi, J., et al. 2010, ApJ, submitted arXiv:0908.3907]

[Hewett \& Wild 2010]Hewett, P.C., \& Wild, V. 2010, MNRAS, in press arXiv:1003.3017]

[Hogg et al. 2001]Hogg, D.W., Schlegel, D.J., Finkbeiner, D.P., \& Gunn, J.E. 2001, AJ, 122, 2129

[Inada et al 2008]Inada, N., et al. 2008, AJ, 135, 496

[Ivezic et al. 2004]Ivezić, Ž., et al. 2004, AN, 325, 583

[Jester et al. 2005]Jester, S., et al. 2005, AJ, 130, 873

[Jiang et al. 2009]Jiang, L., et al. 2009, AJ 138, 305

[Lupton et al. 2001]Lupton, R.H., Gunn, J.E., Ivezić, Ž., Knapp, G.R., Kent, S., \& Yasuda, N. 2001, in ASP Conf. Ser. 238, Astronomical Data Analysis Software and Systems, ed. F.R. Harnden, F.A. Primini, \& H.E. Payne (San Francisco:ASP), 269

[1]Lupton, R.H., Gunn, J.E., \& Szalay, A. 1999, AJ, 118, 1406

[McGreer et al. 2010]McGreer, I.D., et al. 2010, AJ, submitted arXiv:1002.3832]

[Oke \& Gunn 1983]Oke, J.B., \& Gunn, J.E., 1983, ApJ., 266, 713

[Padmanabhan et al. 2008]Padmanabhan, N., et al. 2008, ApJ, 674, 1217

[2]Pier, J.R., Munn, J.A., Hindsley, R.B., Hennessy, G.S., Kent, S.M., Lupton, R.H., \& Ivezić, Ž., 2003, AJ, 125, 1559

[Plotkin et al 2010]Plotkin, R.M., et al. 2010, AJ, 139, 390

[Reyes et al 2008]Reyes, R., et al. 2008, AJ, 136, 2373 
[Richards et al 2001]Richards, G.T., et al. 2001, AJ, 121, 2308

[Richards et al. 2002]Richards, G.T., et al. 2002, AJ, 123, 2945 (GTR02)

[Richards et al. 2003]Richards, G.T., et al. 2003, AJ, 126, 1131

[Richards et al. 2006]Richards, G.T., et al. 2006, AJ, 131, 2766

[Richards et al. 2009]Richards, G.T., et al. 2009, ApJS, 180, 67

[Schlegel et al. 1998]Schlegel, D.J., Finkbeiner, D.P., \& Davis, M. 1998, ApJ, 500, 525

[Schlegel et al. 2009]Schlegel, D., White, M., \& Eisenstein, D.J., 2009, arXiv:0909.4680]

[Schmidt and Green 1983]Schmidt, M., \& Green, R.F. 1983, ApJ, 269, 352

[Schneider et al. 2002]Schneider, D.P., et al. 2002, AJ, 123, 567 (Paper I)

[Schneider et al. 2003]Schneider, D.P., et al. 2003, AJ, 126, 2579 (Paper II)

[Schneider et al. 2005]Schneider, D.P., et al. 2005, AJ, 130, 367 (Paper III)

[Schneider et al. 2007]Schneider, D.P., et al. 2007, AJ, 134, 102 (Paper IV)

[Skrutskie et al 2006]Skrutskie, M.F., et al. 2006, AJ, 131, 1163

[Smith et al. 2002]Smith, J.A., et al. 2002, AJ, 123, 2121

[Stoughton et al. 2002]Stoughton, C., et al. 2002, AJ, 123, 485 (EDR)

[Strateva et al. 2005]Strateva, I.V., Brandt, W.N., Schneider, D.P., Vanden Berk, D.E., \& Vignali, C. 2005, AJ, 130, 387

[Strauss et al. 2002]Strauss, M.A., et al. 2002, AJ, 124, 1810

[Thakar et al 2008]Thakar, A.R., Szalay, A., Fekete, G., and Gray, J. 2008, Computing in Science and Engineering, 10, 30

[Tucker et al. 2006]Tucker, D., et al. 2006, AN, 327, 821

[Vanden Berk et al. 2001]Vanden Berk, D.E., et al. 2001, AJ, 122, 549

[Vanden Berk et al. 2005]Vanden Berk, D.E., et al. 2005, AJ, 129, 2047

[Voges et al. 1999]Voges, W., et al. 1999, A \& A, 349, 389

[Voges et al. 2000]Voges, W., et al. 2000, IAUC, 7432 
[Weinstein et al. 2004]Weinstein, M.A., et al. 2004, ApJS, 155, 243

[3]Wilhite, B.C., et al. 2005, AJ, 633, 638

[Yanny et al. 2009]Yanny, B., et al. 2009, AJ, 137, 4377

[York et al. 2000]York, D.G., et al. 2000, AJ, 120, 1579

[York et al. 2006]York, D.G., et al. 2006, MNRAS, 367, 945 


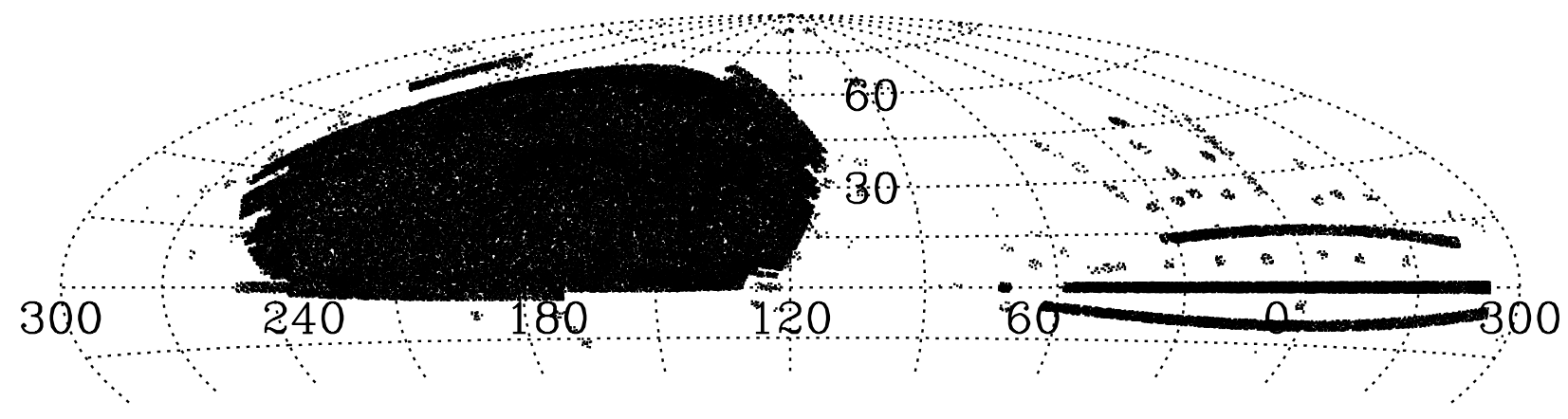

Fig. 1. - The sky distribution of the 105,783 quasars in the catalog in J2000 equatorial coordinates. The primary components of the dataset are those associated with the Legacy Survey (North Galactic Cap, the large contiguous area, and the South Galactic Cap, three narrow stripes aligned with the Celestial Equator) and SEGUE (the points lying off the stripes in the South Galactic Cap). 

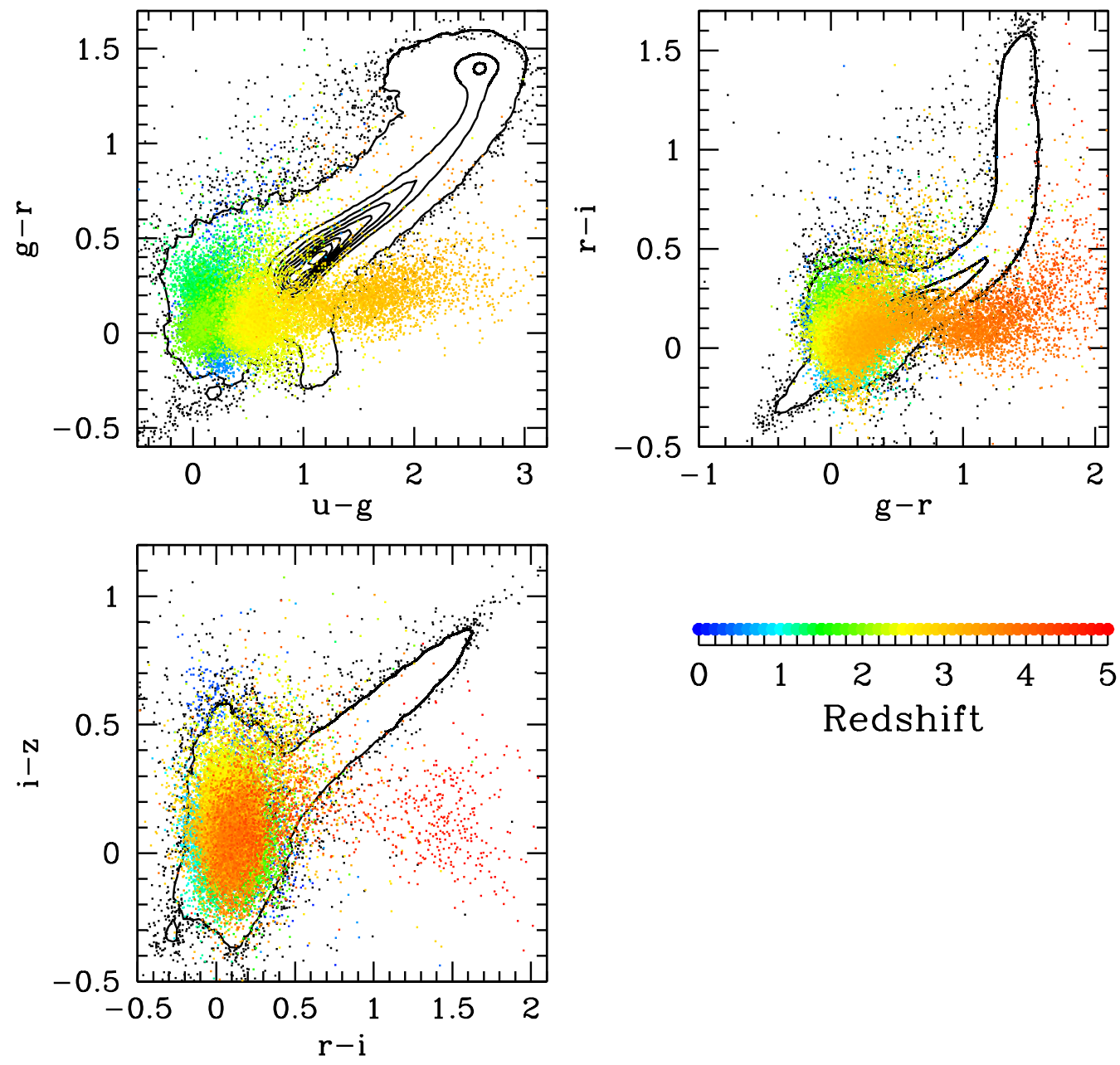

Fig. 2.- The distribution of unresolved sources (black dots and contours) and quasars (color dots) in SDSS ugriz color-color space. The quasars are color-coded by redshift. For clarity we only show $10 \%$ of the $z<2.2$ quasars in the figure. 

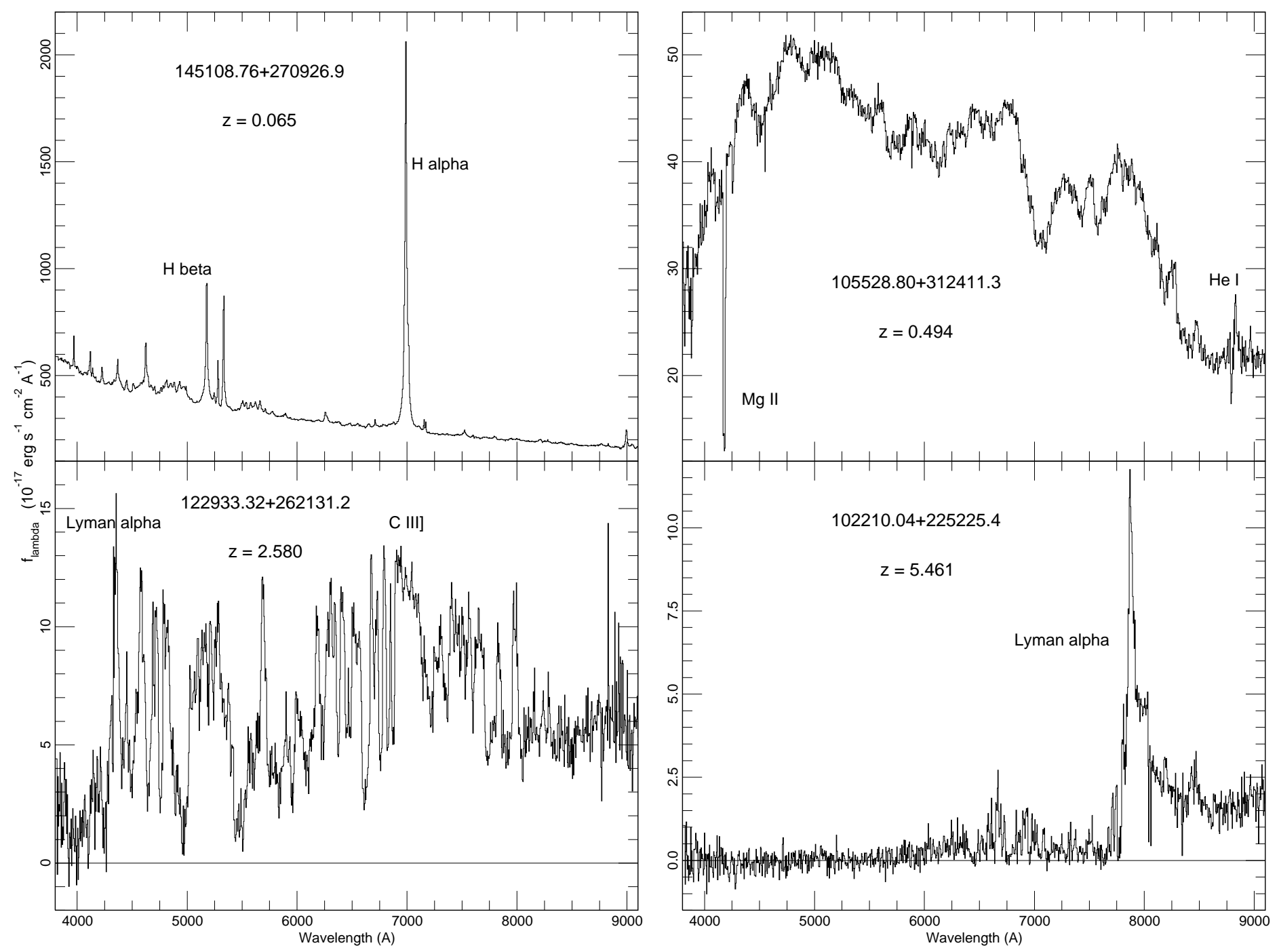

Fig. 3.- SDSS spectra of four quasars that have been obtained since the DR5 catalog. The spectral resolution of the data ranges from 1800 to 2100 . The data have been rebinned to $5 \AA$ pixel $^{-1}$ for display purposes. The panels display (clockwise, starting in upper left) 1) the lowest redshift quasar in the catalog (previously known; PG 1448+273, Schmidt \& Green 1983), 2) a quasar with unusually strong Fe emission (previously known; 2MASS J10552880+3124112, Barkhouse \& Hall 2001), 3) the highest redshift quasar in the catalog (note the nearly complete absorption below the Lyman $\alpha$ emission line and the narrow C IV absorption just longward of the Lyman $\alpha$ emission line), and 4) a striking example of an extreme BAL quasar. 


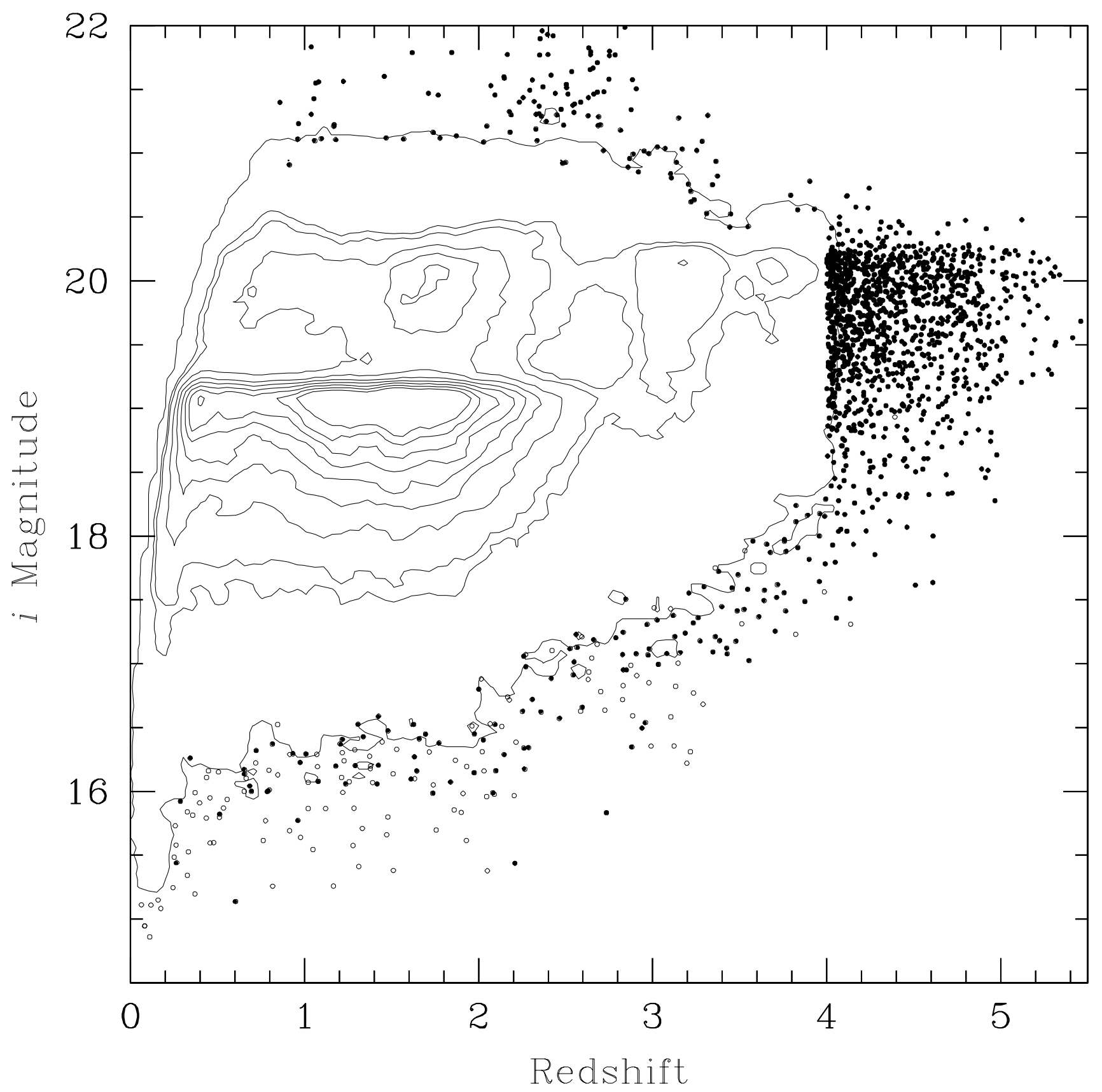

Fig. 4.- The observed $i$ magnitude as a function of redshift for the 105,783 objects in the catalog. Open circles indicate quasars that were recovered, but not discovered, by the SDSS. The distribution is represented by a set of linear contours when the density of points in this two-dimensional space would cause the points to overlap. The steep gradients at $i \approx 19.1$ and $i \approx 20.2$ are due to the flux limit for the targeted low and high redshift parts of the survey; the dip in the counts at $z \approx 2.7$ arises because of the high incompleteness of the SDSS Quasar Survey at redshifts between 2.5 and 3.0 (also see Figure 5). 

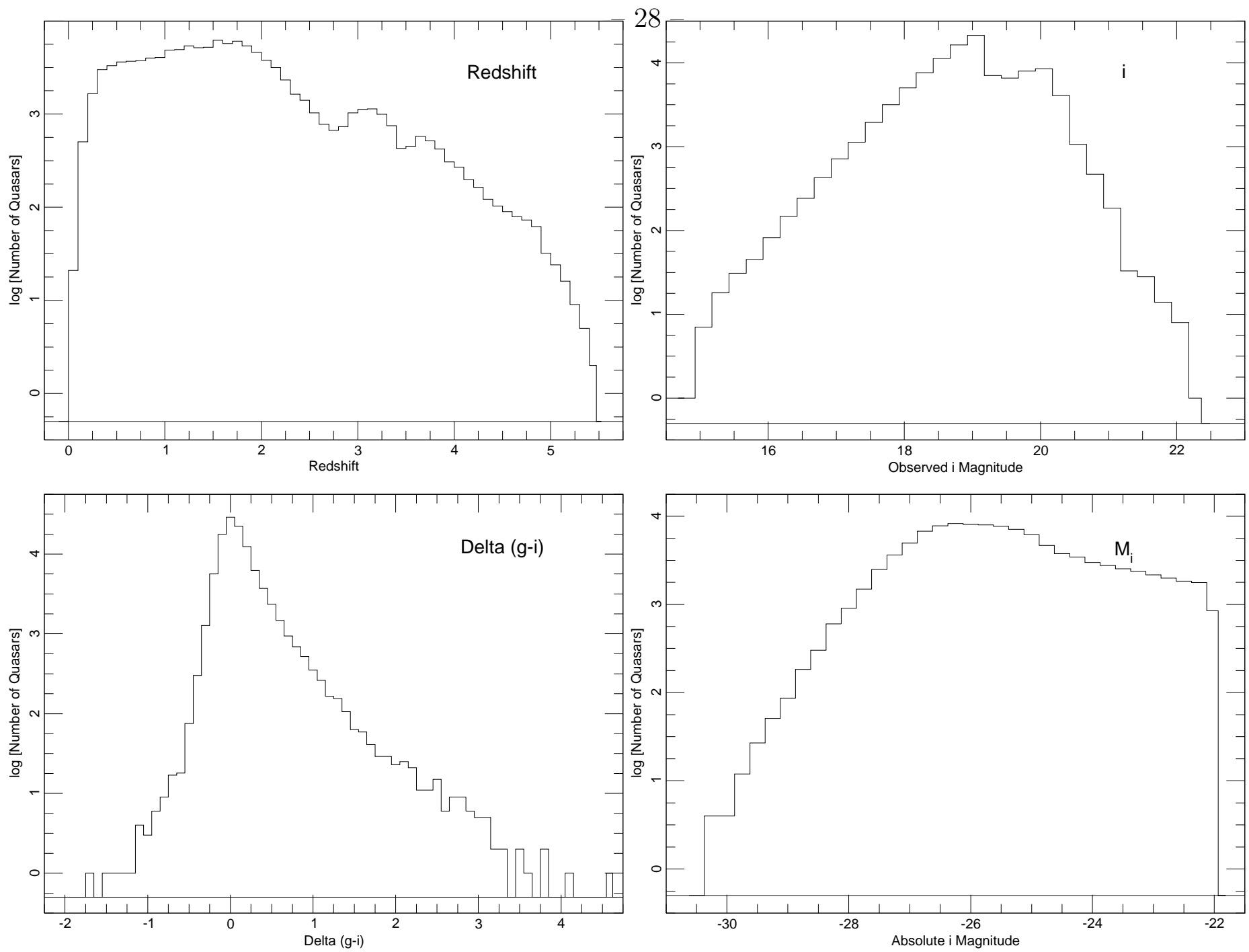

Fig. 5.- The four panels display the distributions of the catalog quasars in redshift, observed $i$ magnitude, $\Delta(g-i)$, and absolute magnitude. The two left panels have bin sizes of 0.1 ; the two panels on the right have magnitude intervals of 0.25 . Logarithmic scales have been adopted because of the enormous dynamic range in all of the histograms. If there are zero points in a bin, the logarithm of the counts is set to -0.3 (horizonal lines in each panel). The redshifts range from 0.065 to 5.46. The dips at redshifts of 2.7 and 3.5 are caused by the reduced completeness of the selection algorithm at these redshifts. The $i$ magnitude in the upper right panel is not corrected for Galactic absorption. The sharp drop that occurs at magnitudes slightly fainter than 19 is due to the flux limit for the low-redshift targeted part of the survey. The SDSS Quasar survey has a bright limit of $i \approx 15.0$ imposed by the need to avoid saturation in the spectroscopic observations. The distribution of the $\Delta(g-i)$ colors shows strong asymmetry, with a tail of objects extending to the red. 

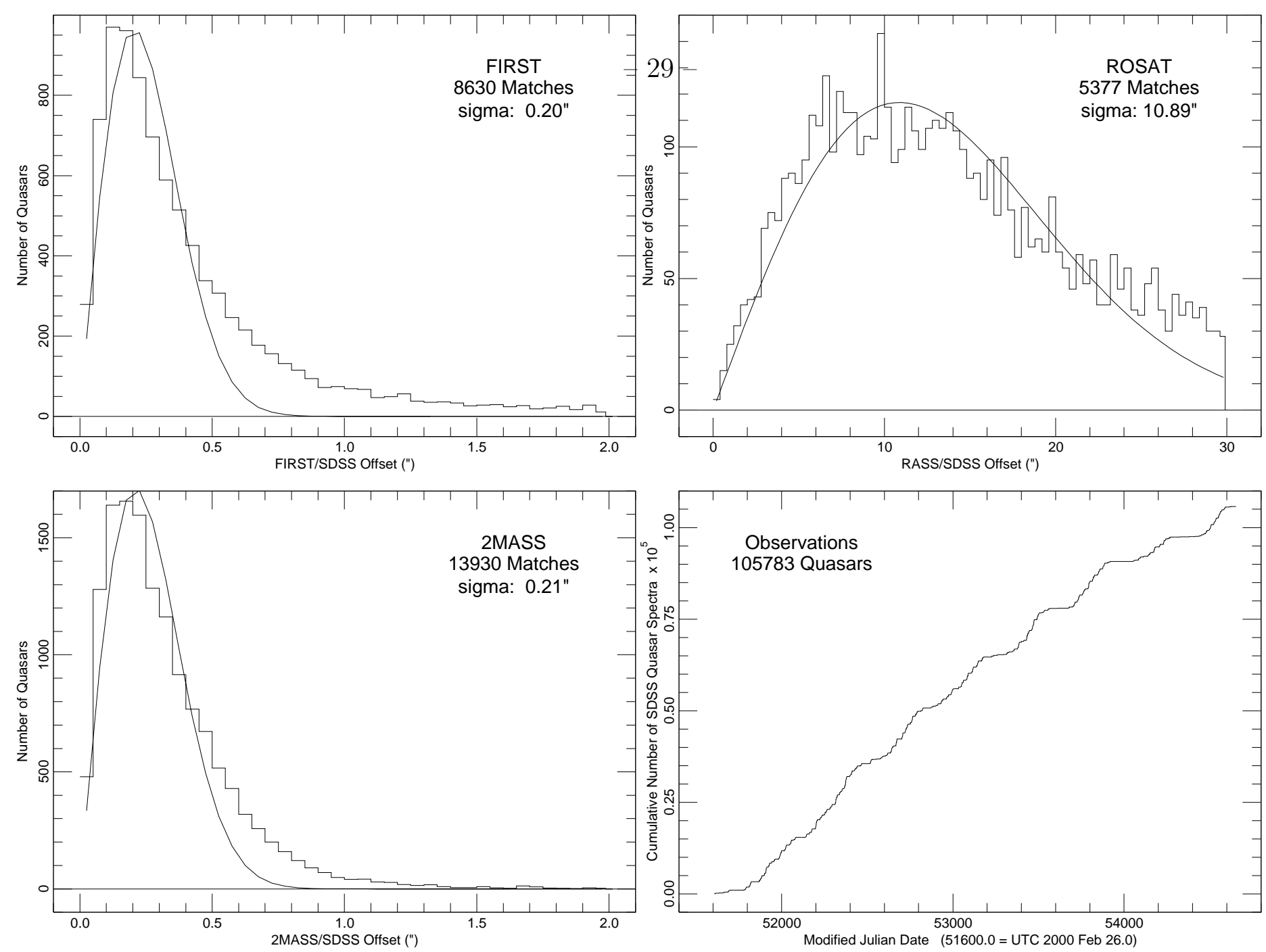

Fig. 6.- The upper row and lower left panels show the distribution of the angular offsets between the SDSS positions and those of the FIRST, ROSAT, and 2MASS coordinates, respectively. The matching radii for the surveys were 2.0" (FIRST), 30.0" (ROSAT), and 2.0" (2MASS). The smooth curves in each of these panels is the expected distribution for a set of matches if the offsets between the objects are described by a Rayleigh distribution; the dispersions of the fit are given in each panel. Only points with separations of less than 1.0" were used in fitting the Rayleigh distribution in the FIRST and 2MASS matching. In the 2MASS case, only points that were actual matches to the published 2MASS catalog are displayed. The Rayleigh distribution is a poor fit to the FIRST and 2MASS matches, as both data sets have a much higher than expected number of matches at radii larger than $\approx 0.5^{\prime \prime}$. The lower right panel displays the cumulative number of DR7 quasars as a function of time; the observations occurred between February 2000 and July 2008. The roughly periodic structure in the curve is produced by the yearly summer maintence schedule and, late in the survey, the limited number of quasar observations during the Fall once that portion of the Legacy Survey spectroscopy was completed. 

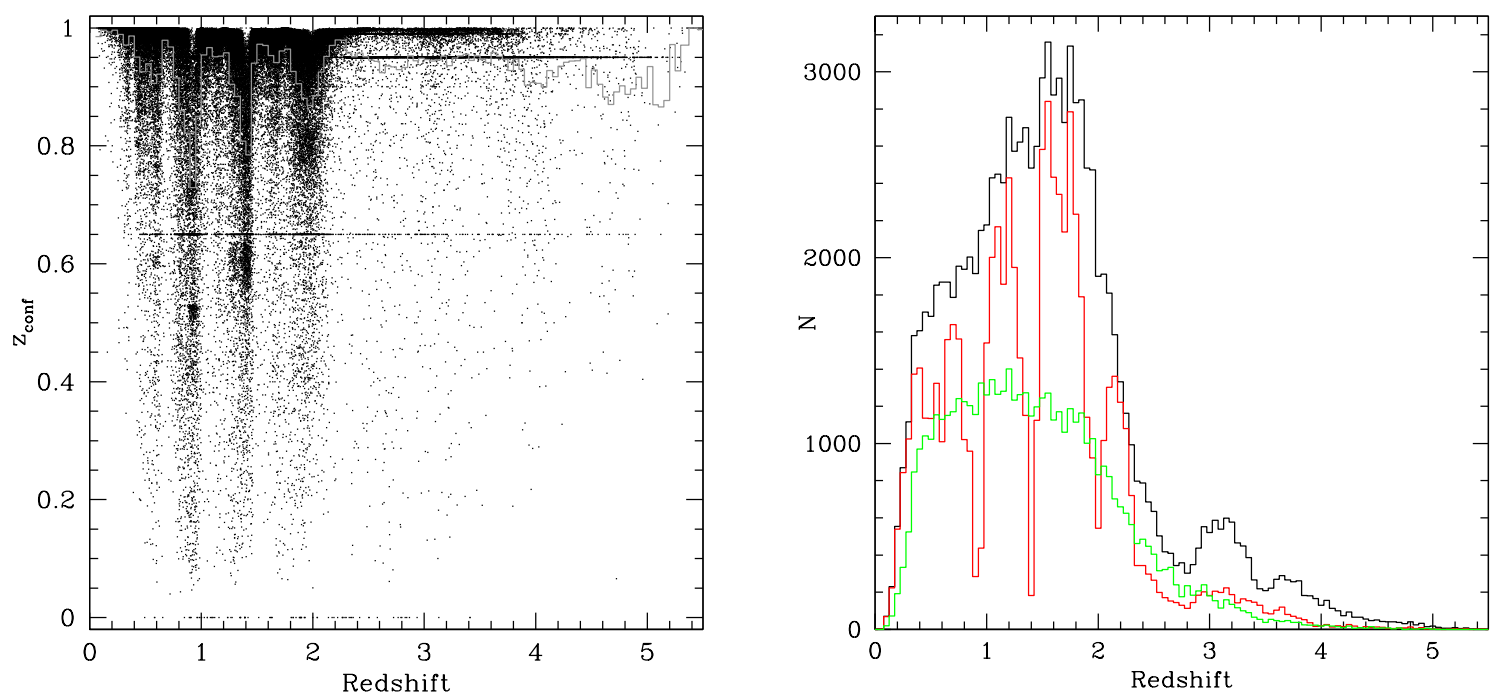

Fig. 7.- Left panel: The distribution in the zconf-redshift plane for the DR7 quasars. Black points indicate individual objects; the gray line shows the moving average in redshift bins of 0.05. Note the banding structure produced by the movement of strong emission lines through the SDSS spectrograph bandpass. A large number of low zconf values occur at the redshifts where there are fewer strong emission features in the SDSS spectra (see text). Right panel: The black histogram shows the raw redshift distribution of the DR7 quasars in the catalog. The red histogram displays the redshift distribution that results from applying a cut of zconf $=0.95$ on the points in the left-hand panel; this selection produces an unphysical redshift distribution. This is not an issue for quasars in this catalog, but must be considered in any attempt to extend the catalog (e.g., to fainter quasars) for the purpose of statistical analyses. The green histogram corrects for the emission-line Malmquist bias and reveals the intrinsic redshift distribution for a fixed continuum luminosity (see text). 
Table 1. SDSS DR7 Quasar Catalog Format

\begin{tabular}{|c|c|c|}
\hline Column & Format & Description \\
\hline 1 & A18 & SDSS DR7 Designation $\quad$ hhmmss.ss+ddmmss.s \\
\hline 2 & F11.6 & Right Ascension in decimal degrees (J2000) \\
\hline 3 & F11.6 & Declination in decimal degrees $(\mathrm{J} 2000)$ \\
\hline 4 & F7.4 & Redshift \\
\hline 5 & F7.3 & BEST PSF $u$ magnitude (not corrected for Galactic extinction) \\
\hline 6 & F6.3 & Error in BEST PSF $u$ magnitude \\
\hline 7 & F7.3 & BEST PSF $g$ magnitude (not corrected for Galactic extinction) \\
\hline 8 & F6.3 & Error in BEST PSF $g$ magnitude \\
\hline 9 & F7.3 & BEST PSF $r$ magnitude (not corrected for Galactic extinction) \\
\hline 10 & F6.3 & Error in BEST PSF $r$ magnitude \\
\hline 11 & F7.3 & BEST PSF $i$ magnitude (not corrected for Galactic extinction) \\
\hline 12 & F6.3 & Error in BEST PSF $i$ magnitude \\
\hline 13 & F7.3 & BEST PSF $z$ magnitude (not corrected for Galactic extinction) \\
\hline 14 & F6.3 & Error in BEST PSF $z$ magnitude \\
\hline 15 & F7.3 & $u$ band Galactic extinction (from Schlegel et al 1998) \\
\hline 16 & F7.3 & $\log N_{H}\left(\log a r i t h m\right.$ of Galactic H I column density in $\left.\mathrm{cm}^{-2}\right)$ \\
\hline 17 & F7.3 & $\begin{array}{l}\text { FIRST peak flux density at } 20 \mathrm{~cm} \text { expressed as AB magnitude; } \\
0.0 \text { is no detection, }-1.0 \text { source is not in FIRST area }\end{array}$ \\
\hline 18 & F8.3 & $\mathrm{S} / \mathrm{N}$ of FIRST flux density \\
\hline 19 & F7.3 & SDSS-FIRST separation in arc seconds \\
\hline 20 & F8.3 & $\log$ RASS full band count rate (counts $\mathrm{s}^{-1}$ ); -9.0 is no detection \\
\hline 21 & F7.3 & $\mathrm{S} / \mathrm{N}$ of RASS count rate \\
\hline 22 & F7.3 & SDSS-RASS separation in arc seconds \\
\hline 23 & F7.3 & $J$ magnitude (2MASS); 0.0 indicates no 2MASS detection \\
\hline 24 & F6.3 & Error in $J$ magnitude \\
\hline 25 & F7.3 & $H$ magnitude (2MASS); 0.0 indicates no 2MASS detection \\
\hline 26 & F6.3 & Error in $H$ magnitude \\
\hline 27 & F7.3 & $K$ magnitude (2MASS); 0.0 indicates no 2MASS detection \\
\hline 28 & F6.3 & Error in $K$ magnitude \\
\hline 29 & F7.3 & SDSS-2MASS separation in arc seconds \\
\hline 30 & I3 & 2MASS Flag $=9 * J$ flag $+3 * H$ flag $+K$ flag \\
\hline
\end{tabular}


Table 1-Continued

\begin{tabular}{|c|c|c|}
\hline Column & Format & Description \\
\hline 31 & $\mathrm{~F} 8.3$ & $\begin{array}{l}\text { Filter flags } 0=\text { no detection, } 1=\text { catalog match, } 2=\text { new photometry } \\
M_{i}\left(H_{0}=70 \mathrm{~km} \mathrm{~s}^{-1} \mathrm{Mpc}^{-1}, \Omega_{M}=0.3, \Omega_{\Lambda}=0.7, \alpha_{\nu}=-0.5\right)\end{array}$ \\
\hline 32 & F7.3 & $\Delta(g-i)=(g-i)-\langle(g-i)\rangle_{\text {redshift }}($ Galactic extinction corrected $)$ \\
\hline 33 & I3 & SDSS Morphology flag $\quad 0=$ point source $\quad 1=$ extended \\
\hline 34 & I3 & SDSS Spectroscopy SCIENCEPRIMARY flag (0 or 1) \\
\hline 35 & I3 & SDSS MODE flag (blends, overlapping scans; 1,2 , or 3) \\
\hline 36 & I3 & Selected with final quasar algorithm (0 or 1$)$ \\
\hline 37 & $\mathrm{I} 12$ & Target Selection Flag (BEST) \\
\hline 38 & I3 & Low-z Quasar selection flag (0 or 1$)$ \\
\hline 39 & I3 & High- $z$ Quasar selection flag (0 or 1 ) \\
\hline 40 & I3 & FIRST selection flag (0 or 1$)$ \\
\hline 41 & I3 & ROSAT selection flag (0 or 1$)$ \\
\hline 42 & I3 & Serendipity selection flag (0 or 1$)$ \\
\hline 43 & I3 & Star selection flag (0 or 1$)$ \\
\hline 44 & I3 & Galaxy selection flag (0 or 1$)$ \\
\hline 45 & I6 & SDSS Imaging Run Number of BEST photometric measurements \\
\hline 46 & I6 & Modified Julian Date of BEST imaging observation \\
\hline 47 & I6 & Modified Julian Date of spectroscopic observation \\
\hline 48 & I5 & Spectroscopic Plate Number \\
\hline 49 & I5 & Spectroscopic Fiber Number \\
\hline 50 & $\mathrm{I} 4$ & SDSS Photometric Processing Rerun Number \\
\hline 51 & I3 & SDSS Camera Column Number (1-6) \\
\hline 52 & I5 & SDSS Field Number \\
\hline 53 & I5 & SDSS Object Identification Number \\
\hline 54 & $\mathrm{I} 12$ & Target Selection Flag (TARGET) \\
\hline 55 & $\mathrm{I} 3$ & Low- $z$ Quasar selection flag (0 or 1$)$ \\
\hline 56 & I3 & High- $z$ Quasar selection flag (0 or 1 ) \\
\hline 57 & I3 & FIRST selection flag (0 or 1 ) \\
\hline 58 & I3 & $R O S A T$ selection flag (0 or 1$)$ \\
\hline 59 & I3 & Serendipity selection flag (0 or 1$)$ \\
\hline 60 & I3 & Star selection flag (0 or 1$)$ \\
\hline
\end{tabular}


Table 1-Continued

\begin{tabular}{ccl}
\hline \hline Column & Format & \\
\hline 61 & I3 & Galaxy selection flag (0 or 1) \\
62 & F7.3 & TARGET PSF $u$ magnitude (not corrected for Galactic extinction) \\
63 & F6.3 & Error in TARGET PSF $u$ magnitude \\
64 & F7.3 & TARGET PSF $g$ magnitude (not corrected for Galactic extinction) \\
65 & F6.3 & Error in TARGET PSF $g$ magnitude \\
66 & F7.3 & TARGET PSF $r$ magnitude (not corrected for Galactic extinction) \\
67 & F6.3 & Error in TARGET PSF $r$ magnitude \\
68 & F7.3 & TARGET PSF $i$ magnitude (not corrected for Galactic extinction) \\
69 & F6.3 & Error in TARGET PSF $i$ magnitude \\
70 & F7.3 & TARGET PSF $z$ magnitude (not corrected for Galactic extinction) \\
71 & F6.3 & Error in TARGET PSF $z$ magnitude \\
72 & I21 & BestObjID (64 bit integer) \\
73 & I21 & SpecObjID (64 bit integer) \\
74 & 1X, A25 & NED Object Name for previously known quasars \\
& & "SDSS" designates previously published SDSS object \\
\hline
\end{tabular}


Table 2. The SDSS Quasar Catalog $\mathrm{V}^{a}$

\begin{tabular}{|c|c|c|c|c|c|c|c|c|c|c|c|c|c|c|}
\hline Object (SDSS J) & R.A. (deg) & Dec (deg) & Redshift & \multicolumn{2}{|c|}{$u$} & \multicolumn{2}{|c|}{$g$} & \multicolumn{2}{|c|}{$r$} & \multicolumn{2}{|c|}{$i$} & \multicolumn{2}{|c|}{$z$} & $A_{2}$ \\
\hline $000006.53+003055.2$ & 0.027228 & 0.515341 & 1.8246 & 20.384 & 0.065 & 20.461 & 0.034 & 20.324 & 0.038 & 20.093 & 0.041 & 20.042 & 0.121 & 0.1 \\
\hline $000008.13+001634.6$ & 0.033900 & 0.276301 & 1.8373 & 20.242 & 0.054 & 20.206 & 0.024 & 19.941 & 0.032 & 19.485 & 0.032 & 19.178 & 0.068 & 0 . \\
\hline $000009.26+151754.5$ & 0.038604 & 15.298477 & 1.1985 & 19.916 & 0.042 & 19.807 & 0.036 & 19.374 & 0.017 & 19.148 & 0.023 & 19.312 & 0.069 & 0.2 \\
\hline $000009.38+135618.4$ & 0.039089 & 13.938450 & 2.2342 & 19.233 & 0.026 & 18.886 & 0.022 & 18.427 & 0.018 & 18.301 & 0.024 & 18.084 & 0.033 & \\
\hline $000009.42-102751.9$ & 0.039271 & -10.464426 & 1.8449 & 19.242 & 0.036 & 19.019 & 0.027 & 18.966 & 0.021 & 18.775 & 0.018 & 18.705 & 0.047 & 0.1 \\
\hline
\end{tabular}

a Table 2 is presented in its entirety in the electronic edition of the Astronomical Journal. A portion is shown here for guidance regarding its form content. The full catalog contains 74 columns of information on 105,783 quasars. 
Table 3. Spectroscopic Target Selection

\begin{tabular}{|c|c|c|c|c|}
\hline Class & $\begin{array}{l}\text { TARGET } \\
\text { Selected }\end{array}$ & $\begin{array}{l}\text { TARGET } \\
\text { Sole } \\
\text { Selection }\end{array}$ & Selected & $\begin{array}{c}\text { BEST } \\
\text { Sole } \\
\text { Selection }\end{array}$ \\
\hline Low- $z$ & 68478 & 22861 & 65823 & 20793 \\
\hline High- $z$ & 24088 & 7789 & 24322 & 6745 \\
\hline FIRST & 5142 & 336 & 5261 & 319 \\
\hline ROSAT & 6302 & 467 & 6403 & 577 \\
\hline Serendipity & 56095 & 19660 & 54478 & 19333 \\
\hline Star & 2338 & 268 & 1194 & 242 \\
\hline Galaxy & 687 & 122 & 754 & 105 \\
\hline None of Above & 5992 & & 10357 & \\
\hline
\end{tabular}


Table 4. Candidate Binary Quasars ${ }^{a}$

\begin{tabular}{ccccccr}
\hline \hline Quasar 1 & Quasar 2 & $i_{1}$ & $i_{2}$ & $z_{1}$ & $z_{2}$ & \multicolumn{1}{c}{$\Delta \theta^{\prime \prime}$} \\
\hline $001201.87+005259.7$ & $001202.35+005313.9$ & 20.0 & 20.2 & 1.652 & 1.637 & 16.0 \\
$011757.99+002104.1$ & $011758.83+002021.4$ & 19.9 & 17.9 & 0.612 & 0.613 & 44.5 \\
$014110.40+003107.1$ & $014111.62+003145.8$ & 20.4 & 20.0 & 1.875 & 1.884 & 42.9 \\
$024511.93-011317.5$ & $024512.12-011314.0$ & 20.2 & 19.4 & 2.462 & 2.460 & 4.5 \\
$025813.65-000326.4$ & $025815.54-000334.2$ & 20.0 & 19.0 & 1.318 & 1.320 & 29.4 \\
$025959.68+004813.6$ & $03000.57+004828.0$ & 19.5 & 16.5 & 0.893 & 0.900 & 19.6 \\
$035053.04-003200.1$ & $035053.29-003114.7$ & 19.5 & 19.3 & 1.993 & 1.995 & 45.5 \\
$074336.85+205512.0$ & $074337.28+205437.1$ & 19.9 & 19.7 & 1.570 & 1.565 & 35.5 \\
$074759.02+431805.3$ & $074759.65+431811.4$ & 19.0 & 19.2 & 0.501 & 0.501 & 9.2 \\
$075715.09+321240.7$ & $075718.02+321320.7$ & 20.6 & 18.4 & 1.446 & 1.458 & 54.6 \\
$082439.83+235720.3$ & $082440.61+235709.9$ & 18.9 & 18.9 & 0.536 & 0.536 & 14.9 \\
$085625.63+511137.0$ & $085626.71+511117.8$ & 18.5 & 19.2 & 0.543 & 0.542 & 21.8 \\
$090923.12+000204.0$ & $090924.01+000211.0$ & 20.0 & 16.4 & 1.882 & 1.866 & 15.0 \\
$094208.81+310736.1$ & $094212.95+310745.5$ & 19.6 & 20.2 & 1.708 & 1.724 & 53.9 \\
$095556.37+061642.4$ & $095559.02+061701.8$ & 17.8 & 20.3 & 1.279 & 1.273 & 44.0 \\
$110357.71+031808.2$ & $110401.49+031817.5$ & 18.2 & 19.1 & 1.941 & 1.922 & 57.3 \\
$110838.31+255521.4$ & $110838.99+255613.2$ & 18.0 & 17.7 & 0.719 & 0.732 & 52.6 \\
$111610.68+411814.4$ & $111611.73+411821.5$ & 19.0 & 18.0 & 3.002 & 2.985 & $13.8^{b}$ \\
$113457.73+084935.2$ & $113459.37+084923.2$ & 18.9 & 19.2 & 1.533 & 1.525 & 27.1 \\
$121840.48+501543.4$ & $121841.00+501535.8$ & 18.3 & 16.8 & 1.458 & 1.455 & 9.1 \\
$122708.94+195751.8$ & $122712.13+195804.2$ & 18.2 & 18.0 & 1.966 & 1.962 & 46.7 \\
$124819.28+370626.3$ & $124823.35+370628.9$ & 19.0 & 19.1 & 1.525 & 1.518 & 48.8 \\
$140012.77+313454.1$ & $140012.85+313452.7$ & 20.0 & 19.6 & 3.317 & 3.316 & $1.7^{c}$ \\
$152107.91+562448.4$ & $152110.12+562436.0$ & 19.1 & 19.8 & 1.578 & 1.566 & 22.2 \\
$155217.93+045646.7$ & $155218.08+045635.2$ & 18.7 & 18.4 & 1.563 & 1.564 & 11.7 \\
$165501.31+260517.4$ & $165502.01+260516.5$ & 17.9 & 17.8 & 1.880 & 1.891 & 9.6 \\
$215727.26+001558.4$ & $215728.35+001545.5$ & 20.5 & 19.2 & 2.570 & 2.553 & 20.8 \\
\hline & & & & & &
\end{tabular}

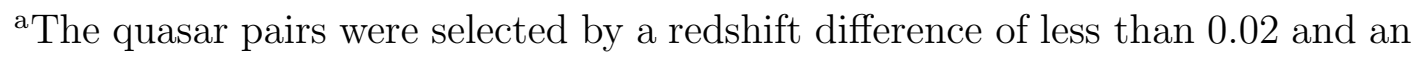
angular separation $(\Delta \theta)$ less than $60^{\prime \prime}$.

${ }^{\mathrm{b}}$ See Hennawi et al. (2010).

${ }^{c}$ Known gravitational lens (Inada et al. 2008). 
Table 5. Quasars Lacking CAS Photometry ${ }^{a}$

\begin{tabular}{|c|c|c|c|c|c|c|c|c|c|c|c|c|c|c|}
\hline Object (SDSS J) & R.A. (deg) & Dec (deg) & Redshift & $u$ & & $g$ & & $r$ & & $i$ & & $z$ & & $A_{u}$ \\
\hline $000439.57-004319.2$ & 1.164883 & -0.722014 & 2.4211 & 21.475 & 0.179 & 20.633 & 0.039 & 20.018 & 0.028 & 19.751 & 0.031 & 19.484 & 0.076 & 0.0 \\
\hline $001509.46+351111.6$ & 3.789439 & 35.186576 & 2.4736 & 20.177 & 0.040 & 19.539 & 0.019 & 19.319 & 0.019 & 19.218 & 0.019 & 18.960 & 0.041 & 0.0 \\
\hline $001510.37+354004.9$ & 3.793231 & 35.668044 & 2.9886 & 20.980 & 0.087 & 19.467 & 0.023 & 19.187 & 0.024 & 19.153 & 0.026 & 19.002 & 0.042 & 0.0 \\
\hline $001552.04+352216.4$ & 3.966839 & 35.371239 & 0.7361 & 19.225 & 0.025 & 19.003 & 0.016 & 19.037 & 0.014 & 19.082 & 0.018 & 18.956 & 0.039 & 0.0 \\
\hline $001628.24+344434.9$ & 4.117698 & 34.743040 & 1.2350 & 18.938 & 0.024 & 18.909 & 0.014 & 18.709 & 0.013 & 18.709 & 0.014 & 18.803 & 0.043 & 0.0 \\
\hline
\end{tabular}

a Table 5 is presented in its entirety in the electronic edition of the Astronomical Journal. A portion is shown here for guidance regarding its form a content. 